East African Medical Journal Vol. 86 No. 10 October 2009

TREATMENT OUTCOME OF TB / HIV POSITIVE AND NEGATIVE SMEAR POSITIVE PULMONARY TUBERCULOSIS PATIENTS TREATED USING DAILY SELF-ADMINISTERED THERAPY IN A CAMEROONIAN DISTRICT HOSPITAL G.E. Sume, MD, MPH, Regional Delegation of Public Health, Littoral Region, International Vaccination Centre, Douala, P.O.Box1115, Douala, Cameroon, M.Hoshen, PhD, Biostatistics, ComputationalBiology Unit, Rosetta Genomics Ltd, 10 PlautSt, Rehovot, Israel, 70706, G. Bita, MD, Regional Delegation of Public Health Littoral, P.O. Box 106, Douala, Cameroon, S. Kabore, MD, MSc, Medical Co-ordinator, Medicins Sans Frontiere Suisse, 1007 Av Agostino Neto, P.O. Box 248, Maputo, Mozambique and V.N. Nzima, MD, Regional Delegation of Public Health, Littoral, Province, P.O. Box 106, Douala, Cameroon

Request for reprints to: Dr. G. E. Sume, Regional Delegation of Public Health Littoral Region, International Vaccination Centre P. O. Box 1115, Douala, Cameroon

\title{
TREATMENT OUTCOME OF TB/HIV POSITIVE AND NEGATIVE SMEAR POSITIVE PULMONARY TUBERCULOSIS PATIENTS TREATED USING DAILY SELF-ADMINISTERED THERAPY IN A CAMEROONIAN DISTRICT HOSPITAL
}

\author{
G.E. SUME, M. HOSHEN, G. BITA, S. KABORE and V.N. NZIMA
}

\begin{abstract}
Background: In our previous study we found that half of the patients treated at the Nylon District Hospital tuberculosis (TB) treatment centre were seropositive. HIV does not only fuel the number of tuberculosis (TB) cases worldwide but it is also at least in part, responsible for the non-achievement of the $85 \%$ cure rate target.

Objective: To compare the TB treatment outcome of SmearPositivePulmonary tuberculosis (SPPT) patients who did an HIV test and those who refused the test as well as compare the treatment outcomes between the HIV positive and HIV negative SPPT patients from 2003 to 2005 , all of whom were treated as outpatients under routine programme conditions. Design: A retrospective study.

Subjects: Four hundred and twenty patients were registered from 2003 to 2005 as having SPPT.

Setting: The Nylon District Hospital, Cameroon.

Results: Thirty five point two per cent of the 386 SPPT patients also had HIV. The overall cure rate, default rate and death rate were $69 \%, 23.6 \%$ and $3.3 \%$ respectively. SPPT/HIV co-infected patients were significantly more likely to default from treatment $\mathbf{2 8 . 7 \%}$ versus $16.8 \%$, OR $1.943,95 \%$ CI 1.150-3.285) to die in the course of treatment $(7.4 \%$ versus $0.4 \%$, OR $23.714,95 \%$ CI 2.894-194.330) or not to be cured (58.8\% versus $78.8 \%$, OR 0.404 , 95\% CI 0.250-0.652) compared to SPPT/HIV negative patients. Likewise SPPT patients not tested for HIV were significantly less cured $(38.2 \%$ versus $71.8 \%$, OR $0.21,95 \% \mathrm{CI}$ $0.099-0.445)$ and defaulted most $(52.9 \%$ versus $21 \%$, OR $4.77395 \%$ CI $2.281-9.991)$ compared to SPPT patients tested for HIV.

Conclusion: SPPT patients infected with HIV or not tested for HIV in the course of TB treatment are likely to suffer from unfavorable treatment outcomes. Thus health personnel prescribing anti- TB drugs should be provided with the necessary expertise to diagnose and manage $\mathrm{HIV}$ so that $\mathrm{TB} / \mathrm{HIV}$ co-infected patients benefit from an integrated package of care in and out of the hospital.
\end{abstract}

\section{INTRODUCTION}

Tuberculosis (TB) disease remains a public health problem worldwide especially in Africa and Asia. Before the advent of Human Immunodeficiency Virus/Acquired Immunodeficiency Syndrome (HIV/AIDS), relatively low disease burden levels were attained by many countries as a result of better treatment outcome. HIV has not only been blamed for the alarming increase of TB cases worldwide but has also been accused of being responsible for the non achievement of the $85 \%$ cure rate target for identified TB cases set by World Health Organisation (WHO) and International Union for the fight Against Tuberculosis and Lung Diseases (IUATLD) (1-3).

While some studies found comparable TB cure rates among HIV positive and HIV negative patients $(4,5)$, others have reported lower cure rates among TB patients infected with $\operatorname{HIV}(6,7)$. On the other hand, higher case fatalities have unanimously been reported in TB / HIV co-infected patients (4-9). The risk of death in HIV positive patients presenting with TB disease 
is approximately four times higher than that of HIV negative TB patients (10). During the initial phase of TB treatment, the cause of death might be due to TB but after the intensive phase of treatment it is usually due to other HIV related complications (4). The one year mortality rate of TB patients infected with HIV ranges from $20-35 \%$ (11).

The impact of HIV on TB treatment outcome in Cameroon is worth to be analysed most especially as the incidence and prevalence of HIV remains high. In 2004, the national programme for the fight against TB reported that $40 \%$ of patients with smear positive pulmonary tuberculosis (SPPT) were HIV positive (12).

The Nylon District Hospital (NDH) with its pioneer HIV/AIDS treatment programme (PREvention et Traitement Intégré du VIh- PRETIVI Project) set up by Médecin Sans Frontiéres/ Switzerland (MSF-CH) and a TB treatment and diagnosis centre provides an ideal setting for the evaluation of the impact of HIV on TB treatment outcome at district level. In this study we compare the TB treatment outcome of SPPT patients who did an HIV test and those who refused the test as well as compare the treatment outcome between the HIV positive and HIV negative SPPT patients from 2003 to 2005, all of whom were treated as outpatients (self-administered drugs) under routine programme conditions. Our findings will provide information on the adequacy of the present treatment strategy as per the National Tuberculosis Programme, at district hospitals or health centre levels where treatment is not daily supervised at least for the intensive phase of therapy, in a country with high HIV burden.

\section{MATERIALS AND METHODS}

Setting: The Nylon District Hospital is a first line referral unit in the Nylon Health District. It also co-habits an HIV/AIDS (PRETIVI) project and a TB diagnosis and treatment centre since 2001. The PRETIVI project was the first of its kind in the Littoral, South West and West regions of Cameroon with over six million inhabitants providing comprehensive care to Persons Living With HIV / AIDS (PLW HIV / AIDS) at district level. The integration of both TB and HIV programmes gave the hospital a good reputation and attracted a lot of patients.

Case detection of tuberculosis is essentially passive. Patients who consulted at the hospital with a dry or productive cough for more than or equal to three weeks with or without chest pain, dyspnoea, haemoptysis, fever, night sweats, weight loss were requested to do a sputum test (three early morning sputum smear in three days). Patients with at least two of the three sputum smear positive for acid fast bacilli were declared SPPT and those who had never taken TB medications were considered as new cases.

Following the national guidelines (12) over the said period, new cases of SPPT were treated with Rifampicine, Isoniazid, Ethambutoland Pyrazinamide (RHEZ) for two months followed by Rifampicine and Isoniazid (RH) for four months on daily basis (2RHEZ/4RH). Relapse sputum smear positive cases (due to treatment failure, defaulting or re-treatment) were treated with Rifampicine, Isoniazid, Ethambutol Pyrazinamide and Streptomycine (RHEZS) for two months then RHEZ for one month followed by Rifampicine, Isoniazid and Ethambutol (RHE) thrice a week for five months (2RHEZS / IRHEZ / $5\left\{\mathrm{RH}_{3} \mathrm{E}_{3}\right\}$ ). Sputum control exams were done for all patients with a positive smear TB at the end of the second (third month for relapse cases) and fifth months of treatment.

HIV test was done in the hospital laboratory on daily basis to patients after precounselling at the psychosocial department. Rapid antibody testing techniques were used in the following pairs with respect to the availability: Determine ${ }^{\circledR}$ HIV-1/ 2 test (ABBOT Diagnostic Division, Hoofddorp, Netherlands) and Immunocomb II Bispot HIV-1/2 (Orgenetics Ltd, Israel) or Determine ${ }^{\circledR} \mathrm{HIV}-1 / 2$ test (ABBOTDiagnostic Division, Hoofddorp, Netherlands) and Camstix HIV 1/2 (Camdiagnostix, Yaounde, Cameroon). A person was considered HIV positive if both tests were positive. Once the first test (Determine ${ }^{\circledR}$ ) was negative, a second test was not done. Indeterminate results were sent to the Centre Pasteur Laboratory in Yaounde for a confirmatory test and the results made known to the patients when available. The results were given to the patients from the 48th hour after post-counselling by workers in the psychosocial department. HIV positive patients were automatically proposed to join the PRETIVI Project during post-counselling. A PRETIVI file was opened for patients with a positive response and he/she referred for medical consultation.

The definitions used for treatmentoutcome by the national guideline are similar to those recommended by WHO and the IUATLD. Treatment outcome was determined after six months for patients with new SPPT and after eight months for relapse cases. In this study the following operational definitions for the given treatment outcome variable were used:

Cured: Patient who presented a negative smear microscopic result at the end of the fifth month of treatment and finished the remaining month(s) of treatment.

Defaulted: Patient who after taking TB medications for one month abandoned for at least two months.

Failed: Patient initially diagnosed with a new case of SPPT in whom acid fast bacilli was still present in his/her fifth month sputum control test.

Died: Patient who died in the course of tuberculosis treatment. 
Transferred out: Patient who started treatment at Nylon District Hospital but was transferred to another tuberculosis treatment centre before the end of his / her treatment.

Completed treatment: Patient who completed his/her $6 / 8$ months TB treatment without presenting his/her 5 th month sputum control test result.

Chronic excretor: Patient diagnosed with relapse SPPT in whom acid fast bacilli was still present in his/her fifth month sputum control test.

Study population and design: It is a retrospective study in which all TB patients with SPPT registered in the Nylon District Hospital centre for the diagnosis and treatment of TB from 2003 to 2005 were included. The TB register was reviewed for age, sex, place of residence, HIV status, current treatment and treatment history.

Data analysis: Data was entered and analysed using SPSS 12.0 for Windows. Logistic regression was used to compare treatment outcomes between patients who did an HIV test and those who did not, as well as between HIV positive and HIV negative patients. Unadjusted and adjusted (for age, gender, health district of residence) Odds Ratios' were reported. The Yates Chi-square tests was used to compare groups of categorical variables. The Fisher exact test (WINPEPI 2007 version 4.1) was also used to analyse the modifying effect of HIV status and treatment history as well as HIV status and health district of residence on treatment outcome. The independent sample t-test was used to compare mean ages.
A 95\% confidence interval was calculated and values of $\mathrm{P}<0.05$ were considered significant.

\section{RESULTS}

A total of 427 patients were registered as having SPPT from 2003 to 2005 . Three patients with combined SPPT and EPT and four patients with inconclusive HIV results were excluded from the analysis. Therefore we present the results of 420 patients.

Patient characteristics: Of the 420 patients included in the analysis, 238(56.7\%) were males. The median age was 30 years (range 13 to 74 years). Only 191(45.5\%) of the patients live in the Nylon Health District. Three hundred and seventy three $(88.8 \%)$ patients were adults aged 15 to 49 years. Up to 386(91.1\%) out of the 420 patients took the HIV test. The patient characteristics of these 386 patients by HIV status are presented in Table 1.

There was no association between HIV status and neither health district of residence nor treatment history. However there was a significant association between HIV status and gender $(P=0.001)$ as well as age group $(\mathrm{P}=0.0003)$. Of the 166 females with SPPT tested for HIV, 75(45.2\%) were HIV positive as opposed to $61(27.7 \%)$ males of the 220 tested, giving a female to male SPPT / HIV co-infection ratio of 1.6. The mean age at diagnosis of patients with SPPT infected with HIV $(33.59 \pm 9.3$ years $)$ is similar $(\mathrm{P}=$ $0.261,95 \% \mathrm{CI}$ of the difference $=-1.008$ to 3.713 ) to that of SPPT patients negative for HIV $(32.24 \pm 12.2$ years). Up to $95(69.9 \%)$ of all SPPT/HIV co-infected patients were aged between 25 to 44 years.

Table 1

Characteristics of SPPT patients by HIV status

\begin{tabular}{|c|c|c|c|c|c|c|}
\hline $\begin{array}{l}\text { Variable and } \\
\text { category }\end{array}$ & $\begin{array}{l}\text { HIV } \\
\text { No. }\end{array}$ & $\begin{array}{l}+(\mathrm{n}=136) \\
(\%)\end{array}$ & $\begin{array}{l}\text { HIV- } \\
\text { No. }\end{array}$ & $\begin{array}{l}(\mathrm{n}=250) \\
(\%)\end{array}$ & $\begin{array}{l}\text { Total per } \\
(\mathrm{T}) \text { category }\end{array}$ & P-value \\
\hline \multicolumn{7}{|l|}{ Gender } \\
\hline Male & 61 & 44.9 & 159 & 63.6 & 220 & \\
\hline Female & 75 & 55.1 & 91 & 36.4 & 166 & 0.001 \\
\hline \multicolumn{7}{|c|}{$\mathrm{HD}$ of residence } \\
\hline Nylon & 54 & 39.7 & 120 & 48 & 174 & \\
\hline Non Nylon & 82 & 60.3 & 130 & 52 & 212 & 0.134 \\
\hline \multicolumn{7}{|c|}{ Treatment history } \\
\hline New case & 107 & 78.7 & 213 & 85.2 & 320 & \\
\hline Old case & 29 & 21.3 & 37 & 14.8 & 66 & 0.12 \\
\hline \multicolumn{7}{|c|}{ Age group (years) } \\
\hline $0-24$ & 25 & 18.4 & 81 & 32.4 & 106 & \\
\hline $25-44$ & 95 & 69.9 & 122 & 48.8 & 217 & \\
\hline $45+$ & 16 & 11.7 & 47 & 18.8 & 63 & 0.0003 \\
\hline
\end{tabular}

P-value from Chi square with continuity correction. HD = Health District 
Treatment outcome: Treatment results of the 420 patients diagnosed with SPPT showed an overall cure rate of $69 \%$, a default rate of $23.6 \%$, a $3.3 \%$ death rate, a $2.9 \%$ treatment completion rate, a $0.7 \%$ treatment failure rate and a transfer out rate of $0.5 \%$ (Table 2 ).

As indicated in Table 3, the cure rate for SPPT cases was $78.8 \%$ for HIV-negative patients and $58.8 \%$ for HIV positive patients. SPPT / HIV co-infected patients were less likely to be cured (OR $0.384,95 \%$ CI 0.250 0.652) as compared to SPPT/HIV negative patients. The default rate for SPPT cases was $28.7 \%$ for HIV- positive patients and $16.8 \%$ for HIV-negative patients. SPPT / HIV co-infected patients were more likely to default(OR1.991, 95\% CI1.150-3.285) thanSPPT / HIVnegative patients. Likewise, the death rate of SPPT cases was 7.4\% for HIV-positive patients and $0.4 \%$ for HIV -negative patients. SPPT/HIV co-infected patients were much more likely to die in the course of treatment [OR 19.759, 95\%CI 2.894-194.330] than SPPT/HIV -negative patients. All these differences remained significant even after adjusting for age, gender, treatment history and residence. Treatment completion, treatment failure and transferred out were not associated with HIV status.

Table 2

Treatment outcome for all patients

\begin{tabular}{|c|c|c|c|c|c|c|c|c|c|c|c|c|c|}
\hline \multirow[t]{2}{*}{ Aspect } & \multicolumn{2}{|c|}{ Total } & \multicolumn{2}{|c|}{ Cured } & \multicolumn{2}{|c|}{$\begin{array}{l}\text { Completed } \\
\text { treatment }\end{array}$} & \multicolumn{2}{|c|}{ Defaulted } & \multicolumn{2}{|c|}{ Died } & \multicolumn{2}{|c|}{ Failed } & $\begin{array}{l}\text { Transferred } \\
\text { out }\end{array}$ \\
\hline & No. & (\%) & No. & (\%) & No. & $(\%)$ & No. & $(\%)$ & No. & $(\%)$ & & $(\%)$ & No. $(\%)$ \\
\hline HIV Tested & 386 & 100 & 277 & 71.8 & 12 & 3.1 & 81 & 21 & 11 & 2.8 & 3 & 0.8 & 0.5 \\
\hline HIV positive & 136 & 100 & 80 & 58.8 & 4 & 2.9 & 39 & 28.7 & 10 & 7.4 & 1 & 0.7 & 1.5 \\
\hline HIV negative & 250 & 100 & 197 & 78.8 & 8 & 3.2 & 42 & 16.8 & 1 & 0.4 & 2 & 0.8 & 0 \\
\hline Not HIV tested & 34 & 100 & 13 & 38.2 & 0 & 0 & 18 & 52.9 & 3 & 8.8 & 0 & 0 & 0 \\
\hline Total & 420 & 100 & 290 & 69 & 12 & 2.9 & 99 & 23.6 & 14 & 3.3 & 3 & 0.7 & 20.5 \\
\hline
\end{tabular}

Table 3

Treatment results for tuberculosis patients by HIV status showing adjusted and unadjusted Odds Ratios

\begin{tabular}{|c|c|c|c|c|c|c|c|c|c|c|c|c|}
\hline \multirow[b]{2}{*}{$\begin{array}{l}\text { Treatment } \\
\text { Results }\end{array}$} & \multirow{2}{*}{\multicolumn{2}{|c|}{$\begin{array}{l}\text { Total } \\
\text { No. }(\%)\end{array}$}} & \multirow{2}{*}{\multicolumn{2}{|c|}{$\begin{array}{l}\text { HIV+ } \\
\text { No. }(\%)\end{array}$}} & \multirow{2}{*}{\multicolumn{2}{|c|}{$\begin{array}{l}\text { HIV- } \\
\text { No. }(\%)\end{array}$}} & \multicolumn{3}{|c|}{ Non adjusted } & \multirow[b]{2}{*}{$\mathrm{OR}^{*}$} & \multicolumn{2}{|c|}{ Adjusted $^{* *}$} \\
\hline & & & & & & & $\mathrm{OR}^{*}$ & P-value & $95 \% \mathrm{CI}$ & & P-value & $95 \% \mathrm{CI}$ \\
\hline Cured & 277 & 71.8 & 80 & 58.8 & 197 & 78.8 & 0.384 & $<0.0001$ & $0.243-0.607$ & 0.404 & 0.0002 & $0.250-0.652$ \\
\hline $\begin{array}{l}\text { Completed } \\
\text { treatment }\end{array}$ & 12 & 3.1 & 4 & 2.9 & 8 & 3.2 & 0.917 & 0.889 & $0.271-3.101$ & 0.691 & 0.57 & $0.193-2.474$ \\
\hline Defaulted & 81 & 21 & 39 & 28.7 & 42 & 16.8 & 1.991 & 0.007 & $1.210-3.276$ & 1.943 & 0.013 & $1.150-3.285$ \\
\hline Died & 11 & 2.8 & 10 & 7.4 & 1 & 0.4 & 19.759 & 0.005 & $2.502-156.104$ & 23.714 & 0.003 & 2.894-194.330 \\
\hline Failed & 3 & 0.8 & 1 & 0.7 & 2 & 0.8 & 0.919 & 0.945 & $0.083-10.223$ & 0.657 & 0.749 & $0.051-8.553$ \\
\hline $\begin{array}{l}\text { Transferred } \\
\text { out }\end{array}$ & 2 & 0.5 & 2 & 1.5 & 0 & 0.0 & * & * & * & * & * & * \\
\hline Total & 386 & 100 & 136 & 100 & 250 & 100 & & & & & & \\
\hline
\end{tabular}

${ }^{*} \mathrm{OR}$ of treatment outcomes for SPPT cases for HIV-positive relative to HIV-negative patients.

**Adjusted for age, gender, health district of residence and treatment history 
Table 4

Treatment results for tuberculosis patients by HIV test acceptability showing adjusted and unadjusted Odds Ratios

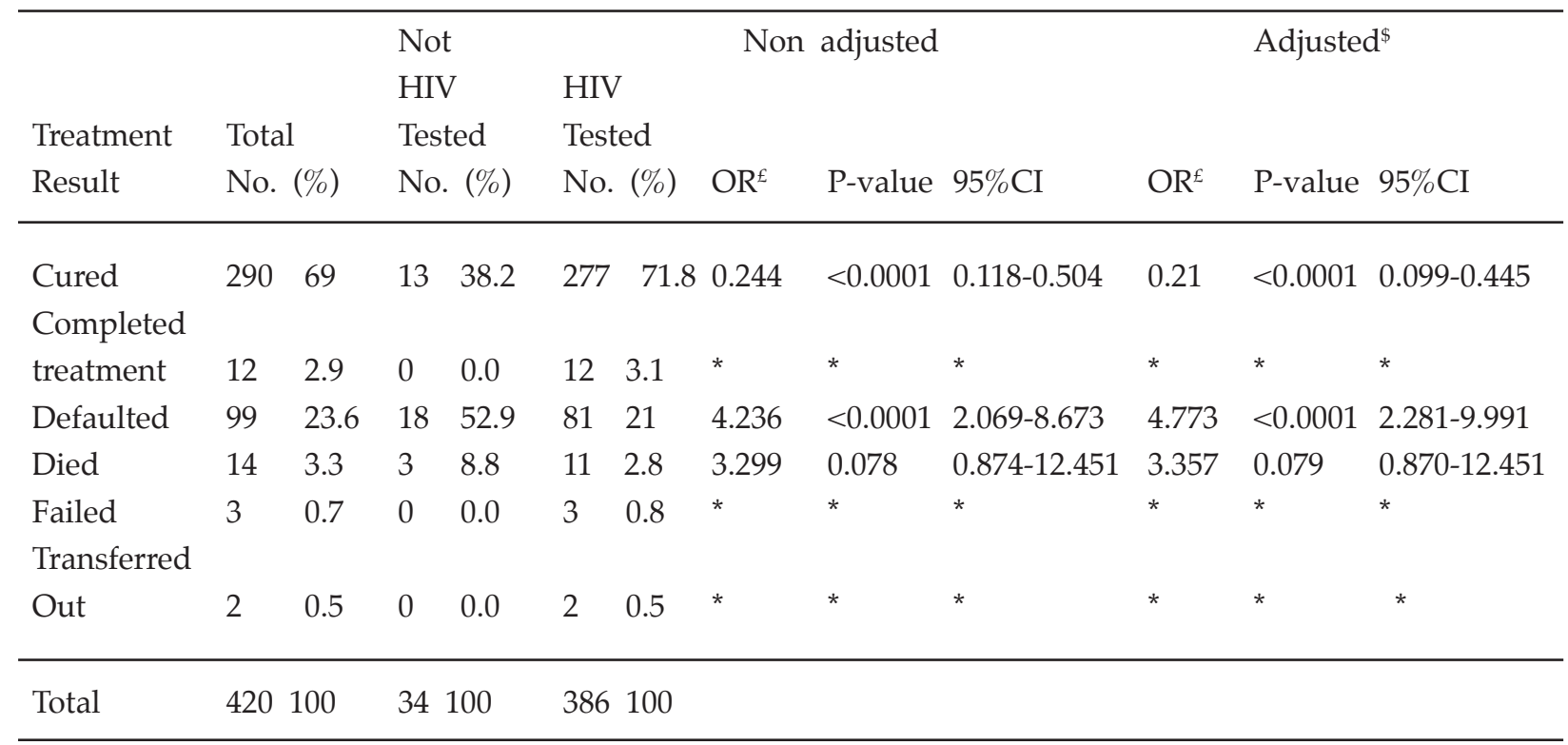

EOR of treatment outcomes for SPPT cases for non HIV tested patients relative to HIV tested patients. ${ }^{\$}$ Adjusted for age, gender, health district of residence and treatment history

Treatment outcome was associated with HIV status for SPPT patients resident in Nylon Health District $(\mathrm{P}=0.0196)$ as well as non residents $(\mathrm{P}=0.0002)$.

The cure rate $(63 \%$ versus $56.1 \%)$, default rate (25.9\% versus $30.5 \%)$ death rate $(5.6 \%$ versus $8.5 \%)$ and treatment failure rate $(0 \%$ versus $1.2 \%)$ for SPPT/HIV positive patients resident in NHD was more favorable compared to the same group of non Nylon residents respectively.

Likewise treatment outcome of SPPT was associated with HIV status both for new cases of TB $(\mathrm{P}=0.0006)$ and for cases of relapse $(\mathrm{P}=0.0056)$. The cure rate $(59.8 \%$ versus $55.2 \%)$ and death rate $(6.6 \%$ versus $10.3 \%)$ in co-infected new SPPT cases was more favorable compared to relapse co-infected SPPT cases. Meanwhile co-infected relapse patients had a better outcome when it came to default rate $(27.6 \%$ versus $29 \%)$ and treatment failure $(0 \%$ versus $0.9 \%)$.

The cure rate for SPPT patients tested for HIV was $71.8 \%$ as opposed to $38.2 \%$ for SPPT patients not tested for HIV. SPPT patients not tested for HIV were less likely to be cured [OR 0.244, 95\% CI 0.0990.445] compared to SPPT patients tested for HIV. The default rate for SPPT patients was $21 \%$ for those tested for HIV and $52.9 \%$ for those not tested for HIV. SPPT patients not tested for HIV were more likely to default [OR 4.236, 95\% CI 2.281-9.991] than those tested. After adjusting for age, sex, residence and treatment history, the differences in cure rate and default rate remained significant (Table 4). The other treatment outcomes were not significantly associated with HIV test acceptability.

\section{DISCUSSION}

The prevalence of HIV infection among SPPT patients in our study was $35.2 \%$. We found that in patients with SPPT, HIV status was associated with gender and age. Women and adults aged 25 to 45 years were the mostSPPT / HIV co-infected. The overall cure rate, default rate and death rate were $69 \%, 23.6 \%$ and $3.3 \%$ respectively. SPPT/HIV co-infected patients were significantly more likely to default from treatment, to die in the course of treatment or not to be cured compared to SPPT / HIV negative patients. Likewise SPPT patients not tested for HIV were significantly less cured and defaulted most compared to SPPT patients tested for HIV.

According to the third Demographic Health Survey (DHS III) of 2004 in Cameroon, the prevalence of HIV in the general population increases progressively from the age of 15 years and reaches a maximum of $10.3 \%$ in women aged 25 to 39 years and $8.6 \%$ in men aged 35 to 39 years (13). These age groups are amongst the most co-infected in our study. Also, the modifying effect of HIV to the TB-gender association is also related to the higher prevalence of HIV infection in women $(6.8 \%)$ as opposed to men $(4.1 \%)$ in the general population as reported in the DHS III. Therefore, supporting the fact that HIV is a very potent risk factor for $\mathrm{TB}$, either by increasing the risk of direct progression of primary disease to full blown disease or increasing the risk of reactivating latent bacilli (14).

The female to male SPPT / HIV co-infection ratio of 1.6 in our study is similar to the female: male HIV 
ratio of 1.7 in the general population as in the DHS III (13). Other common grounds between our TB/HIV co-infection study and the HIV study of the DHS III are the similar age group distribution in SPPT/HIV coinfection and HIV infection in the general population and the female dominance in HIV infection as well as SPPT/HIV infection. Thus, in a country where surveys are scarcely done, a reflection needs to be carried out on the use of SPPT / HIV co-infection rate as a proxy for the estimation of HIV prevalence in some areas of the country.

The overall cure rate of $69 \%$ in our study is slightly higher than the $61 \%$ obtained by Banerjee and colleagues in Ntcheu District hospital in Malawi (7) but lower than the $76 \%$ found by Van den Broek et al in Mwanza Tanzania (6) and $77 \%$ by EI Sony et al in Sudan (5). This may be due to the fact that we used self-administered treatment as from day one except in severely ill patients who were treated as in-patients and discharged as soon as they got better. All the cited studies treated their patients with DOTS during the intensive phase and then ambulatory treatment during the continuation phase. The significantly higher cure rate of SPPT/HIV negative patients (but lower than the recommended $85 \%$ ) compared to SPPT/HIV positive ones in our study was common to all the above settings and of almost similar proportions (5-7). Meanwhile Murray and colleagues (4) studying miners in South Africa found similar cure rates between TB/HIV positive $(89 \%)$ and TB/HIV negative $(88 \%)$ patients under very strict, regular follow-up and well organised programme conditions after excluding death cases.

Tuberculosis patients tested for HIV had a far better outcome compared to their non tested counterparts because they benefited from an integrated care package especially those with a positive result. The non tested ones either were very sick to be counselled, presented at a terminal phase or refused the test. As such they died in the course of treatment or defaulted because they did not offer the team the opportunity to manage them in their entirety. Similar findings were reported by Banerjee and colleagues in Malawi (7).

Our overall death rate of $3.3 \%$ is very small just as the default rate of $23.6 \%$ is very high compared with other studies $(4,6,7)$. This is probably due to an underestimation of the number of deaths. We did not put in place a system to identify the residence of patients or to actively search for those who abandoned their treatment. Most of the known dead cases die in the hospital for one reason or another. It is thus likely that a good proportion of the defaulted patients are likely dead cases. We also confirmed the high case fatalities in TB / HIV co-infected patients earlier reported by many authors (4-9).
Nylon residents had a better outcome because the HIV programme ran in the same hospital at the beginning would preferably enroll HIV positive patients living within the district and refer the others to the nearest treatment centres around their residents. Hence Nylon patients started benefiting from integrated care early enough while the others had to spend further time to adapt and be incorporated to other treatment sites. Distance would not explain the difference because locomotion is not a problem in a metropolitan city like Douala.

Strengths and limitations: This paper is a follow-up to our previous publication on the prevalence of the HIV virus infection among tuberculosis patients in the Nylon District Hospital tuberculosis treatment centre (15). It goes further to emphasize the rates of the various TB treatment outcomes in HIV positive versus HIV negative patients and HIV tested versus HIV non tested patients. It also brings forth the importance of an integrated patient care approach in TB and HIV programmes in order to attain the 85\% cure rate target recommended by WHO.

Our data did not permit us to do survival analysis which would have permitted us to better appreciate the treatment outcome especially the mortality rates of the various groups. Also, the number of cases for some treatment outcome categories were small giving very wide or incalculable confidence intervals.

DOTS per say wasn't used in our setting because of the absence of the necessary logistics and human resources. Nonetheless an analysis to determine when patients abandoned treatment showed that there was no significant difference in the number of patients who defaulted before (intensive phase) or after (continuation phase) their first sputum control test (at the end of the second month of treatment).

In conclusion, SPPT/HIV co-infected patients significantly die more in the course of treatment, default more from treatment and are less likely to be cured from TB compared to SPPT / HIV negative patients. In the same way, SPPT patients not tested for HIV are significantly less cured and defaulted most compared to SPPT patients tested for HIV. In this light we therefore recommend that, not only should a system of active search for lost to follow-up patients be put to place, health personnel prescribing anti- TB drugs be provided with the necessary expertise to diagnose and manage HIV so that TB/HIV co-infected patients benefit from an integrated package of care in and out of the hospital. TB patients who refuse to do the HIV test or whose state of health does not permit them to be counselled should be considered HIV positive until proven otherwise. 


\section{ACKNOWLEDGEMENT}

This work was conducted during the MPH studies of G.E. Sume in the Legacy Heritage International Master of Public Health Programme at the Hebrew University Hadassah Braun School of Public Health and Community Medicine, Jerusalem, Israel, with scholarship support from the Pears Foundation (UK).

\section{REFERENCES}

1. Chum, H.J., Obrien, R.J., Chond, T.M., et al. An epidemiological study of tuberculosis and HIV infection in Tanzania, 1991-1993. AIDS. 1996; 10: 299-309.

2. Murray, J.F. Tuberculosis and HIV infection: global perspectives. Respiratory. 1997; 2: 209-213.

3. Lucas, S.B., Hounnou, A., Peacock, C., et al. The mortality and pathology of HIV infection in a West African city. AIDS. 1993; 7: 1675-1679.

4. Murray, J., Sonnenberg, P., Shearer, S.C. and GodfreyFaussett, P. Human immunodeficiency virus and the outcome of treatment for new and recurrent pulmonary tuberculosis in African patients. Am. J. Respir. Crit. Care. Med. 1999; 159:733-740.

5. El-Sony, A.I., Khamis, A.H., Enarson, D.A., et al. Treatment results of DOTS in 1797 Sudanese tuberculosis patients with or without HIV coinfection. Int. J. Tuberc. Lungs. Dis. 2002; 6: 1058-1066.

6. Van den Broek, J., Mfinanga, S., Moshiro, C. et al. Impact of human immunodeficiency virus infection on the outcome of treatment and survival of tuberculosis patients in Mwanza, Tanzania. Int. J. Tuberc. Lung. Dis. 1998; 2: 547-552.

7. Barberjee, A., Moyo, S., Salaniponi, F. and Harries, A.D.HIV testing and tuberculosis treatment outcome in a rural district in Malawi. Trans. R. Soc. Trop. Med. Hyg. 1997; 91: 707-708.
8. Winqvist, N., Naucler, A., Gomes, V., et al. Three year follow-up of patients with pulmonary tuberculosis in Guinea-Bissau, West Africa. Int. J. Tuberc. Lung. Dis. 2000; 4: 845-852.

9. Malkin, E., Prazuck, T., Simonnet, F., et al. Tuberculosis and human immunodeficiency virus infection in West Burkina Faso: clinical presentation and clinical evolution. Int. J. Tuberc. Lung. Dis. 1997; 1: 68-74.

10. Stoneburner, R., Laroche, E., Prevots, R., et al. Survival in a cohort of human immunodeficiency virus-infected tuberculosis patients in New York city. Implications for the expansion of the AIDS case definition. Arch. Intern. Med. 1992; 152: 2033-2037.

11. Gustafson, P., Gomes, V.F., Vieira, C.S., et al. Clinical predictors for death in HIV-positive and HIV-negative tuberculosis patients in Guinea-Bissau. Infection. 2007; 35: 69-80.

12. Programme Nationale de Lutte contre la Tuberculose, Cameroun. Guide Technique pour les personnels de sante. 2nd ed. Yaounde. Union International Contre la Tuberculose et les Maladies Respiratoires; 2004. p.18-19.

13. Institut National de la Statistique (INS) et ORC Macro. 2004. Enquete Demographique et de la Sante du Cameroun 2004. Calverton, Maryland, USA: INS et ORC Macro.2004; 294-315.

14. Daley, C. L., Small, P. M., Schecter, G. F., et al. An outbreak of tuberculosis with accelerated progression among persons infected with the human immunodeficiency virus. An analysis using restriction-fragment-length polymorphisms. N. Engl. J. Med. 1992; 326: 231-235.

15. Sume, G.E., Etogo, D., Kabore, S., et al. Seroprevalence of human immunodeficiency virus infection among tuberculosis patients in the Nylon Health District Hospital tuberculosis treatment centre. East Afr. Med. J. 2008; 85: 529-536. 